\title{
Understanding High-Utilizing Patients Based on Social Risk Profiles: a Latent Class Analysis Within an Integrated Health System
}

\author{
Artair Rogers, $M S^{1,2,3}$ (D) , Yi R. Hu, MS ${ }^{l}$, Adam Schickedanz, MD, PhD ${ }^{4}$, \\ Laura Gottlieb, $M D, M P H^{5}$, and Adam Sharp, $M D, M S^{6,7}$
}

${ }^{7}$ Kaiser Permanente Southern California, Pasadena, CA, USA; ${ }^{2}$ Health Leads, Boston, MA, USA; ${ }^{3}$ Health Leads, Los Angeles, CA, USA; ${ }^{4}$ Department of Pediatrics, David Geffen School of Medicine at UCLA, Los Angeles, CA, USA; ${ }^{5}$ Department of Family and Community Medicine, University of California, San Francisco, San Francisco, CA, USA; ${ }^{\circ}$ Research and Evaluation Department, Kaiser Permanente Southern California, Pasadena, CA, USA; ${ }^{7}$ Department of Emergency Medicine, Kaiser Permanente Los Angeles Medical Center, Los Angeles, CA, USA.

J Gen Intern Med 35(7):2214-6

DOI: $10.1007 / \mathrm{s} 11606-019-05510-9$

(c) Society of General Internal Medicine 2020

\section{INTRODUCTION}

Data on chronic conditions and clinical diagnoses is used to predict patient outcomes and utilization of health services and subsequently shapes clinical care. ${ }^{1}$ Despite strong associations between social risks and health outcomes, little is known about advancing social care practices in healthcare settings. This study explores how social risks cluster by type and frequency to better understand how patterns of social risks may be associated with chronic conditions and healthcare utilization with the aim to improve care for patients and communities.

\section{METHODS}

We utilized prospectively collected social risk data gathered from a social needs intervention for adult predicted healthcare high utilizers and applied latent class analysis (LCA) based on 14 patient-reported social risks: food insecurity, healthy food, housing, housing safety, employment, transportation, financial security, utility assistance, public benefits, financial counseling, affordable medical care, health literacy, caregiver support, and social support. ${ }^{2}$ LCA is a probabilistic approach that empirically identifies clusters of individuals by categorical variables using a maximum likelihood approach. Tests of model fit were used to indicate the optimal number of distinguishable classes. After comparing with Pearson's $\chi^{2}$, likelihood ratio $\chi^{2}$, Akaike's information criterion, and LoMendell-Rubin's likelihood ratio test, the lowest Bayesian information criterion (BIC) evaluated the best LCA model fit. $^{3}$ After creating the classes, we used patient data from claims and our electronic health record to describe each cluster. Tests of proportions and Student's $t$ tests compared

Received September 23, 2019

Revised September 23, 2019

Accepted October 18, 2019

Published online January 9, 2020 proportions and means by latent class. The study was approved by the Kaiser Permanente Southern California Institutional Review Board.

\section{RESULTS}

Our sample included 2533 high-utilizing patients who completed screening, of which $1984(65 \%)$ reported at least one social risk. The best-fitting LCA model categorized individuals into four classes (BIC $=28,171.96$ ).

Classes differed by types and overall number of social risks as well as race and insurance carrier (Table 1). Yet, the number of social risks per patient differentiated classes most. All of class 1 possessed 4 or more risks while a majority of class 4 had no self-reported risk $(p<0.001)$ (Table 2$)$.

The class with the most social risks (class 1) had the highest total utilization. Compared with the lowest social risk class (class 4), patients in class 1 had significantly more total (5.5 vs. $4.3, p=0.003)$, emergency department ( 2.1 vs. $1.2, p<$ $0.001)$, and inpatient visits ( 1.5 vs. $1.1, p<0.001)$ despite fewer medical comorbidities based on the Charlson score (Table 2). Additionally, patients in class 1 more often identified as black compared with those in class 4 (35\% vs. $13 \%, p<$ $0.001)$

\section{DISCUSSION}

Our study found that in a predicted high healthcare utilization patient sample, latent classes emerge based on endorsed social risks. The class with the most social risks had the highest utilization of care, despite being younger and having the fewest medical comorbidities. Patterns of social risks appear to provide valuable, complementary information to the clinical variables traditionally used to predict utilization. ${ }^{4}$

Often, social care interventions are based on clinical diagnosis, age, or insurance coverage instead of the types or number of social risks, which our findings indicate should influence the design of these interventions. ${ }^{5}$ Furthermore, this study suggests that the number and types of social risks may 
Table 1 Patient Characteristics by Latent Class

\begin{tabular}{|c|c|c|c|c|c|c|c|}
\hline & $\begin{array}{l}\text { Patient } \\
\text { characteristics }\end{array}$ & $\begin{array}{l}\text { Total } \\
(N=2533)\end{array}$ & $\begin{array}{l}\text { Class } 1 \\
(N=421) \\
\text { Most social } \\
\text { risk factors }\end{array}$ & $\begin{array}{l}\text { Class } 2 \\
(N=320)\end{array}$ & $\begin{array}{l}\text { Class } 3 \\
(N=439)\end{array}$ & $\begin{array}{l}\text { Class } 4 \\
(N=1353) \\
\text { Fewest social } \\
\text { risk factors }\end{array}$ & $\begin{array}{l}p \text { value for } t \text { test for } \\
\text { difference between class } 1 \\
\text { and class } 4\end{array}$ \\
\hline \multirow{14}{*}{$\begin{array}{l}\text { Mean } \\
\text { (SD) } \\
\text { Percent } \\
(N)\end{array}$} & Age & $66.1(15.0)$ & $60.5(15.1)$ & $62.9(15.2)$ & $66.4(15.1)$ & $68.5(14.4)$ & $p<0.001$ \\
\hline & Gender & & & & & & \\
\hline & Male & $61(1555)$ & $57(242)$ & $51(163)$ & 66 (289) & $64(862)$ & $p=0.01$ \\
\hline & Female & 39 (978) & $43(179)$ & $49(157)$ & $34(150)$ & $36(491)$ & \\
\hline & Race/ethnicity & & & & & & \\
\hline & Asian & $4.3(110)$ & $4(17)$ & $4(13)$ & $6(27)$ & $4(52)$ & $p=0.79$ \\
\hline & Black & $20.9(530)$ & $35(148)$ & $26(82)$ & $29(126)$ & $13(174)$ & $p<0.001$ \\
\hline & Hispanic & $26.3(667)$ & $34(145)$ & 35 (111) & $22(97)$ & 23 (309) & $p=0.16$ \\
\hline & White & $46(1153)$ & $24(99)$ & $33(105)$ & $39(172)$ & $58(782)$ & $p<0.001$ \\
\hline & Insurance & & & & & & \\
\hline & Medicaid only & $4(101)$ & $8.6(36)$ & $7.8(25)$ & $3.4(15)$ & $1.8(25)$ & $p=0.009$ \\
\hline & Medicare only & $58.3(1476)$ & $48.0(202)$ & $56.3(180)$ & $56.7(249)$ & $62.5(845)$ & $p<0.001$ \\
\hline & Dual & $4.4(111)$ & $8.3(35)$ & $6.9(22)$ & $4.8(21)$ & $2.4(33)$ & $p<0.001$ \\
\hline & Commercial & $33.4(845)$ & $35.2(148)$ & $29.1(93)$ & $35.1(154)$ & $33.3(450)$ & $p=0.472$ \\
\hline \multirow{15}{*}{$\begin{array}{l}\text { Percent } \\
(N)\end{array}$} & Social risk by domain & & & & & & \\
\hline & Food insecurity & $27.5(697)$ & $87.8(370)$ & $0.8(3)$ & $0.1(0)$ & $0.9(12)$ & $p<0.001$ \\
\hline & Healthy food & $28.6(724)$ & $89.4(376)$ & $80.6(258)$ & $13.2(58)$ & $1.9(25)$ & $p<0.001$ \\
\hline & $\begin{array}{l}\text { Homelessness/safe } \\
\text { place }\end{array}$ & $8.9(225)$ & $39.2(165)$ & $6.0(19)$ & $8.2(36)$ & $0.3(3)$ & $p<0.001$ \\
\hline & Housing conditions & $9.4(238)$ & $32.0(135)$ & $7.9(25)$ & $14.2(62)$ & $0.8(11)$ & $p<0.001$ \\
\hline & Adult education & $4.6(116)$ & $18.6(78)$ & $2.4(8)$ & $5.2(23)$ & $0.5(6)$ & $p<0.001$ \\
\hline & $\begin{array}{l}\text { Transportation } \\
\text { (medical) }\end{array}$ & $20.1(508)$ & $48.2(203)$ & $24.1(77)$ & $34.6(152)$ & $4.6(63)$ & $p<0.001$ \\
\hline & Financial insecurity & $34.3(870)$ & $97.6(411)$ & $64.1(205)$ & $46.0(202)$ & $2.3(31)$ & $p<0.001$ \\
\hline & Utility assistance & $22.6(573)$ & $76.7(323)$ & 33.4 (107) & 27.0 (119) & $1.0(14)$ & $p<0.001$ \\
\hline & Public benefits & $10.4(264)$ & $40.1(169)$ & $7.7(25)$ & $14.1(62)$ & $0.3(4)$ & $p<0.001$ \\
\hline & Financial counsel. & $7.4(188)$ & $30.5(128)$ & $1.9(6)$ & $11.4(50)$ & $0.0(0)$ & $p<0.001$ \\
\hline & $\begin{array}{l}\text { Financial } \\
\text { assist-medical care }\end{array}$ & $19.3(488)$ & $53.8(226)$ & 34.2 (109) & 25.7 (113) & $2.1(28)$ & $p<0.001$ \\
\hline & Health literacy* & $15.4(389)$ & $36.4(153)$ & $15.9(51)$ & $21.1(92)$ & $6.4(87)$ & $p<0.001$ \\
\hline & $\begin{array}{l}\text { Caregiver } \\
\text { assistance } * *\end{array}$ & $11.3(285)$ & $17.9(76)$ & $6.2(20)$ & $21.3(94)$ & $6.6(90)$ & $p<0.001$ \\
\hline & Social isolation*** & $21.9(554)$ & $54.7(230)$ & $24.1(77)$ & 34.9 (153) & $6.0(81)$ & $p<0.001$ \\
\hline
\end{tabular}

*Health literacy includes all positive responses: all, most, some

** Only $12 \%$ of pilot population identified as caregivers of an individual who is physically or mentally disabled

***Social isolation includes all positive responses: always, often, sometimes

Table 2 Healthcare Utilization, Comorbidity Score, and Number of Social Risks per Patient by Latent Class

\begin{tabular}{|c|c|c|c|c|c|c|c|}
\hline & & $\begin{array}{l}\text { Total } \\
(N=2533)\end{array}$ & $\begin{array}{l}\text { Class 1 } \\
(N=421) \\
\text { Most social } \\
\text { risk factors }\end{array}$ & $\begin{array}{l}\text { Class 2 } \\
(N=320)\end{array}$ & $\begin{array}{l}\text { Class 3 } \\
(N=439)\end{array}$ & $\begin{array}{l}\text { Class } 4 \\
(N=1353) \\
\text { Fewest social } \\
\text { risk factors }\end{array}$ & $\begin{array}{l}p \text { value for test for difference } \\
\text { between class } 1 \text { and class } 4\end{array}$ \\
\hline \multirow[t]{4}{*}{$\begin{array}{l}\text { Mean } \\
\text { (SD) }\end{array}$} & $\begin{array}{l}\text { Utilization } \\
\text { Total } \\
\text { utilization }\end{array}$ & $4.5(6.7)$ & $5.5(8.4)$ & $5.0(6.0)$ & $3.8(5.0)$ & $4.3(6.7)$ & $p=0.003$ \\
\hline & $\begin{array}{l}\text { ED } \\
\text { utilization }\end{array}$ & $1.4(2.8)$ & $2.1(4.3)$ & $1.8(2.7)$ & $1.2(1.7)$ & $1.2(2.4)$ & $p<0.001$ \\
\hline & $\begin{array}{l}\text { IP } \\
\text { utilization }\end{array}$ & $1.2(1.6)$ & $1.5(2.1)$ & $1.3(1.6)$ & $1.1(1.4)$ & $1.1(1.5)$ & $p<0.001$ \\
\hline & $\begin{array}{l}\text { OP } \\
\text { utilization }\end{array}$ & $1.9(5.1)$ & $1.8(4.8)$ & $1.9(4.7)$ & $1.5(4.0)$ & $2.0(5.6)$ & $p=0.509$ \\
\hline \multirow{6}{*}{$\begin{array}{l}\text { Mean } \\
\text { (SD) } \\
\text { Percent } \\
(N)\end{array}$} & $\begin{array}{l}\text { Charlson } \\
\text { score }\end{array}$ & $7.0(3.1)$ & $6.7(3.1)$ & $7.1(3.2)$ & $7.1(3.0)$ & $7.1(3.1)$ & $p=0.021$ \\
\hline & $\begin{array}{l}\text { Social risks pe } \\
\text { No social } \\
\text { risk }\end{array}$ & $\begin{array}{l}\text { patient } \\
34(851)\end{array}$ & $0(0)$ & $0(0)$ & $0(0)$ & $63(851)$ & $p<0.001$ \\
\hline & 1 social risk & $15(380)$ & $0(0)$ & $0(0)$ & $0(1)$ & $28(379)$ & $p<0.001$ \\
\hline & $\begin{array}{l}2 \text { social } \\
\text { risks }\end{array}$ & $10(254)$ & $0(0)$ & $11(34)$ & $25(110)$ & 8 (110) & $p<0.001$ \\
\hline & $\begin{array}{l}3 \text { social } \\
\text { risks }\end{array}$ & $8(206)$ & $0(0)$ & $23(75)$ & 27 (119) & $1(12)$ & $p=0.039$ \\
\hline & $\begin{array}{l}4+\text { social } \\
\text { risks }\end{array}$ & $33(842)$ & $100(421)$ & $66(211)$ & $48(210)$ & $0(0)$ & $p<0.001$ \\
\hline
\end{tabular}


have as much utility in predicting health outcomes and service utilization as the traditional clinical variables. We found disparities in social risks for racial/ethnic minorities, women, and among Medicaid enrollees. Therefore, addressing equity in the design and implementation of healthcare-based social care interventions is essential to maximize impact. ${ }^{6}$

These descriptive results should inform future efforts to develop predictive algorithms that incorporate social risk data and spur more research on strategies for using social risk data to advance social care practices in healthcare settings.

Corresponding Author: Artair Rogers, MS; Health Leads, Los Angeles, CA, USA

\section{REFERENCES}

1. Sundararajan V, Henderson T, Perry C, Muggivan A, Quan H, Ghali WA. New ICD-10 version of the Charlson comorbidity index predicted in- hospital mortality. J Clin Epidemiol. 2004;57(12):1288-1294. https://doi. org/10.1016/j.jclinepi.2004.03.012.

2. Schickedanz A, et al. Impact of Social Needs Navigation on Utilization Among High Utilizers in a Large Integrated Health System: A QuasiExperimental Study. J Gen Intern Med. 2019; https://doi.org/10.1007/ s11606-019-05123-2.

3. Nylund K, Asparouhov T, Muthen B. Deciding on the Number of Classes in Latent Class Analysis and Growth Mixture Modeling: A Monte Carlo Simulation Study. Struct Equation Model Multidiscip J. 2007; 14(4): 535569. https://doi.org/10.1080/10705510701575396.

4. Chen J, Ellis RP, Toro KH, Ash AS. Mispricing in the Medicare Advantage Risk Adjustment Model. INQUIRY J Health Care Organ, Provision, Financing. 2015;52:004695801558308. https://doi.org/10.1177/ 0046958015583089.

5. Norris S, Nichols P, Caspersen $\mathbf{C}$, et al. The effectiveness of disease and case management for people with diabetes. A systematic review. Am J Prev Med. 2002;22(4):15-38. https://doi.org/10.1016/s0749-3797(02) 00423-3.

6. Camargo KRD. Closing the gap in a generation: Health equity through action on the social determinants of health. Glob Public Health. 2011;6(1):102-105. https://doi.org/10.1080/17441692.2010.514617.

Publisher's Note Springer Nature remains neutral with regard to jurisdictional claims in published maps and institutional affiliations. 